\title{
Lusotopie
}

Recherches politiques internationales sur les espaces

issus de l'histoire et de la colonisation portugaises

$\mathrm{XV}(1) \mid 2008$

Indiens du Mozambique et d'Afrique orientale

\section{Contribution à l'étude des sentiments identitaires des indiens de Madagascar}

\author{
Ludovic Gandelot
}

\section{OpenEdition \\ Journals}

\section{Édition électronique}

URL : http://journals.openedition.org/lusotopie/823

ISSN : 1768-3084

Éditeur :

Association des rechercheurs de la revue Lusotopie, Brill, Karthala

Édition imprimée

Date de publication : 30 juin 2008

Pagination : 175-182

ISSN : 1257-0273

\section{Référence électronique}

Ludovic Gandelot, «Contribution à l'étude des sentiments identitaires des indiens de Madagascar », Lusotopie [En ligne], XV(1) | 2008, mis en ligne le 09 mars 2016, consulté le 19 avril 2019. URL : http:// journals.openedition.org/lusotopie/823 


\section{CONTRIBUTION À L'ÉTUDE DES SENTIMENTS IDENTITAIRES DES INDIENS DE MADAGASGAR}

L'article présente la minorité indienne de Madagascar sous un double regard, relatif au développement de ses activités économiques et à ses sentiments identitaires communautaires, à l'égard de la France et de la Grande Île. En tentant de protéger leur héritage culturel, les communautés gujaraties préservent un système social qui a pu les rendre d'autant plus impopulaires que leur pouvoir économique s'agrandissait. C'est autour de ce qui peut apparaître comme un paradoxe que la question indienne à Madagascar peut être appréhendée, d'apparentes permanences ne devant pas occulter la fluidité des allégeances.

\section{Contributo para o estudo dos sentimentos identitários dos Indianos de Madagáscar}

$\mathrm{O}$ artigo apresenta a minoria indiana de Madagáscar sob um duplo olhar, relativo ao desenvolvimento das suas actividades económicas e aos seus sentimentos identitários, relativamente à França e à "Grande Île ». Ao tentarem proteger a sua herança cultural, as comunidades gujarati preservam um sistema social que pôde torná-las tanto mais impopulares que o seu poder económico aumentava. É à volta do que pode aparecer como um paradoxo que a questão indiana pode ser apreeendida, com permanências aparentes que não devem ocultar a fluidez dos laços de fidelidade.

\section{Contribution to studies of feelings of identity among the Indians of Madagascar}

This article provides a dual insight into the Indian minority in Madagascar, looking into the development of its economic activities and its feelings of community identity, in relation to France and the Grande Ile itself. In attempting to protect their cultural heritage, the Gujarati communities have preserved a social system that has made them increasingly unpopular as their economic power has grown. It is through what could be seen as a paradox that the Indian issue in Madagascar can be understood, for apparent continuities must not hide shifting allegiances.

$\mathrm{D}$ epuis la fin du $x^{e}$ siècle jusqu'à nos jours, les Indiens ont formé, à Madagascar, un ensemble démographiquement marginal. Ils étaient environ 7000 en $1926^{1}, 10000$ en $1948^{2}$, 13000 en $1955^{3}$ et 20000 en 19994. Ceci provient

${ }^{1}$ Gouvernement Général de Madagascar et déPendances, Annuaire général, [Paris], 1948, Imprimerie officielle, Tananarive, 1926.

${ }^{2}$ Demaille, La question hindoue à Madagascar. Rapport de mission parlementaire, Centre des archives d'outre-mer (CAOM, Aix-en-Provence), Fonds Ministériels, séries géographiques, Madagascar (FM/SG/MAD), c. 437, d. 1198.

${ }^{3}$ Anonyme, La question indienne à Madagascar, [1955 ou 1956], CAOM, Fonds Territoriaux, Madagascar, Gouvernement général de Madagascar (FT/MAD/GGM), 6(5) D41.

${ }^{4}$ N. Razafimandimby, "Communauté indienne 0,2 \% de la population, $15 \%$ du PIB », Revue de l'Océan Indien (Madagascar), juin 1999: 25-28. 
notamment du fait, qu'à une très grande majorité, ils se sont installés à Madagascar en intégrant librement des réseaux de commerce anciens qui trouvent dans le phénomène colonial un nouveau moyen d'expansion. Mais dans l'ensemble des réseaux migratoires gujaratis, Madagascar reste une terre lointaine et l'Afrique de l'Est réserve à la même époque de nombreuses opportunités commerciales. La structure du groupe est, elle aussi, particulière dans la mesure où tous sont originaires du Gujarat au nord-ouest de l'Inde et en particulier du Cutch et du Kathiawar (régions littorales du Gujarat). À ce titre, malgré quelques différences dialectales, ils parlent tous la même langue. Le groupe se divise en cinq communautés socioreligieuses. On trouve une minorité hindouiste, autour de $10 \%$, qui présente la particularité d'être arrivée plus tardivement que les autres; des musulmans sunnites en même proportion; des Bohras, shiites ismailis dawoudis et des Khojas, eux-mêmes divisés en deux entités distinctes à partir du dernier tiers du $\mathrm{xx}^{\mathrm{e}}$ siècle ${ }^{5}$ : d'un coté, des shiites ismaïlis nizarites agakhanis et de l'autre, des shiites ithna asheri, duodécimains).

Il y aurait grande pertinence à étudier ces différences communautaires, fondées tant sur les plans religieux que migratoire. Cependant, ayant fait le choix d'aborder ici principalement le groupe des Khojas, on étudiera surtout ce qui les lie : la question des constructions identitaires issues de l'expérience migratoire, de l'installation et du développement des activités économiques.

\section{Des migrations à l'installation à Madagascar}

La venue à Madagascar d'Indiens originaires du Gujarat résulte d'un long processus aux origines incertaines. Si des contacts semblent attestés dès le $\mathrm{xvI}^{\mathrm{e}}$ siècle $^{6}$, ce n'est qu'à partir du xIX $^{e}$ que l'on discerne le processus historique conduisant à la fixation des Indiens sur l'île. En 1826, les tentatives infructueuses du sieur Lyell, en vue de créer une agence commerciale à Majunga (dans le nord-ouest), témoignent non seulement de l'implication des Gujaratis dans le commerce de cette région, mais aussi de leurs efforts pour empêcher la concurrence ${ }^{7}$. D'après le même auteur, ils sont alors déjà connus sous le nom de karana ${ }^{8}$. Grandidier voyageant en Août 1869 dans les baies de Mourousang et de Narinda dit des Betsimisaraka (population de l'Est), qui viennent faire un peu de commerce, qu'ils sont appelés tsikarankarana par les Sakalavas qui habitent la région : ils ne sont pas Indiens musulmans, karana, mais font comme eux'. L'emploi et la déformation du

${ }^{5}$ Voir sur ce sujet L. GAndelot, "Islam shia ithna asheri et migrations chez les Khojas, 1860-1925 ", in M. Boivin (ed.), Les Ismailiens d'Asie du Sud: gestion des héritages et productions identitaires, Paris, L'Harmattan, à paraître.

${ }^{6}$ S. Blanchy, Karana et Banians, Paris, L'Harmattan, 1995 : 45-47.

${ }^{7}$ Voir C. Allibert \& F. Verin, «Deux voyages inédits sur Madagascar et les Comores autour de 1840, J.S. Leigh et J. Marshall », Centre d'Étude et de Recherche sur l'océan Indien (CEROI-Inalco, Paris), 1991: 69 et 72 (« Travaux et documents », 15).

${ }^{8}$ Parfois considéré comme une altération du mot Coran, le terme semble bien vouloir dire, au regard des témoignages des Indiens eux-mêmes, comptable.

${ }^{9}$ A. Grandidier, Notes et carnets de voyage, Paris, Muséum d'Histoire naturelle, Fonds Grandidier B 17. Il s'agit des notes manuscrites prises par Alfred Grandidier lors de son voyage à Madagascar, juin 1868-juillet 1870. Informations recueillies par Klara Boyer-Rossol que je remercie ici vivement. 
terme pour signifier une réalité à la fois semblable et différente, puisque les deux groupes font du commerce, confortent dans l'idée que la présence des Indiens dans cette partie de l'île est déjà ancienne.

C'est aussi l'époque où les réseaux migratoires et commerciaux des Khojas, centrés sur le Gujarat et Zanzibar, s'intensifient. À l'image des autres communautés commerçantes d'origine gujaratie (Parsis, Bhatias, Bohras, Memons et Khojas), les Khojas sont encore en situation de migrations temporaires mais déjà plus dans celui du voyage saisonnier où les commerçants restent quelques semaines avant de repartir. La création d'agences sur la côte ouest du territoire témoigne du passage à une autre étape du processus migratoire. Même s'il faut reconnaître que l'on manque encore de sources pour l'étude de cette époque, tout au moins pour les datations en amont, on peut assurer qu'à ce stade le temps du séjour peut durer quelques années. Ce passage intermédiaire entre le voyage saisonnier et la structuration en communauté concevant son avenir à Madagascar, dure approximativement jusqu'aux années 1930. En 1926, les statistiques de l'Annuaire de Madagascar témoignent à la fois de la surproportion masculine (1 homme pour 0,53 femmes) mais également de l'importance numérique de la classe d'âge des moins de 15 ans (ils comptent pour la moitié de l'ensemble). Selon d'autres sources $^{10}$, jusqu'en 1939, 200 à 250 nouveaux venus viennent s'installer chaque année à Madagascar. Il s'agit en ces années surtout de femmes, de jeunes venus retrouver leurs parents après avoir étés éduqués au Gujarat et probablement d'Indiens hindouistes $^{11}$

\section{Les Indiens et la colonie à la fin des années 1940}

Entre l'instauration de la colonie en 1896 et la Seconde Guerre mondiale, les activités indiennes se concentrent sur le commerce des articles importés par les grandes compagnies européennes et la collecte des produits naturels.

La pénétration dans les villages des côtes nord et ouest est telle que dans certaines régions comme Morondava (approximativement au milieu de la côte ouest), la quasi-totalité du commerce de la première décennie du $\mathrm{xx}^{\mathrm{e}}$ siècle est opérée par les Indiens. Face à l'inquiétude de quelques traitants français ${ }^{12}$, l'administration

10 Demaille, La question hindoue..., op. cit. : 34.

11 Représentant $10 \%$ de la minorité indienne, les Hindous restent une communauté peu étudiée ayant fait souche assez tardivement à partir d'Ambato-Boeni (d'après Pieraly Remtoulla, communication personnelle, Paris, septembre 2007). Mais d'autres hindous gujaratis d'Afrique du Sud ont côtoyé Madagascar, fuyant la guerre des Boers, et l'ont quitté au début du $\mathrm{xx}^{\mathrm{e}}$ siècle. Cf Nanji Kalidas Menta, Dream Half expressed, an autobiography, Bombay, G.U Mehta, 1955.

12 Dans une lettre au gouvernement général, L. Samat, autrefois grand commerçant de Morondava dans l'ouest de l'île, s'exprime en ces termes : «Leur manière, leur mode de commerce est un problème que je n'ai pas pu résoudre. Voyant cela, et avec les occupations que j'ai [il s'est mis au service de l'administration coloniale], j'ai cessé depuis quelques années toute sorte de commerce. », CAOM, Fonds Madagascar, série 2D, province de Morondava, $\mathrm{n}^{\circ} 171,1896$. 
ne peut, elle, que conclure d'une façon générale aux bienfaits de cette situation. Les Indiens contribuent largement en effet au développement, notamment économique, de la colonie. Au début de la période coloniale, certaines personnalités khojas entretiennent de très bonnes relations avec les administrations civile et militaire. Ils approvisionnent l'armée, contribuent à la connaissance du pays, font leur possible pour trouver des engagés, coolies employés aux grands travaux d'aménagements (port, voirie de la ville de Majunga). Vers 1925, la scierie des frères Ramjee de Morondava distribue du bois de construction jusque dans le nord de l'île. Leur réseau de distribution, maritime et fluvial, contribue aussi à désenclaver certaines régions de la côte ouest ${ }^{13}$. Du point de vue fiscal, ils payent non seulement les taxes de séjour mais aussi des droits de patentes supérieurs à ceux des Européens ${ }^{14}$. Ils s'imposent parfois aussi en relais de l'administration coloniale en payant les impôts de leurs employés ${ }^{15}$.

L'administration coloniale n'est pas la seule à profiter de la présence des Gujaratis. Les compagnies françaises vivent aussi sur le réseau des boutiquiers et collecteurs indiens qui s'infiltrent au plus profond des campagnes malgaches. Plus, ils facilitent les migrations ${ }^{16}$ car les Indiens acceptant, à la différence des colons français, les conditions de vie des villages reculés, participent au succès des grossistes des compagnies françaises.

Jusqu'après la guerre donc, la présence des Indiens, malgré la récurrence de pratiques commerciales parfois douteuses (défauts de comptabilité, faillites frauduleuses) ne fait pas vraiment l'objet d'attentions particulières. Ce n'est que sous le gouvernement général de $\mathrm{P}$. de Chevigné, et en plein mouvement nationaliste et insurrectionnel, que l'enrichissement considérable et illégal de certaines familles d'origine gujaratie est sévèrement combattu. Quelques années plus tard, certains milieux malgaches s'inquiètent dans la presse (relayée en métropole et dans l'océan Indien) de ce qui est jugé comme une plaie pour le pays ${ }^{17}$. Il faut dire que pendant les années de guerre, le marché noir est florissant et la corruption généralisée ${ }^{18}$. Ceci est confirmé dans les faits puisque le haut-commissaire doit se défaire du chef de la province de Majunga ${ }^{19}$ ainsi que du chef de district de Maevatanana.

${ }^{13}$ Pendant la Seconde Guerre mondiale, l'administration des Comores reconnaît même que son ravitaillement est entièrement dépendant des navires indiens.

${ }^{14}$ Les droits de patentes supplémentaires pour les commerçants asiatiques et européens sont en vigueur depuis 1904 et sont augmentés en 1923 (arrêté du 24 octobre). On trouvera dans le dossier D754 des Archives nationales de Madagascar, Antananarivo, Madagascar, les documents relatant la légitimation de cette mesure et la défense des intérêts indiens par le gouvernement britannique.

${ }_{15}$ Ainsi en est-il de la famille Ramjee de Morondava déjà citée. «Ils ont payés depuis de longues années, les impôts des milliers d'indigènes travaillant dans leurs chantiers ", notes de renseignements du Commissaire divisionnaire, Morondava, 1938, Archives nationales de Madagascar, Antananarivo, Madagascar, Dossier D738.

${ }^{16}$ Demaille, op. cit.

17 Voir les articles de G. Razafintsalama, in Fandrosoana-Baovao (Tananarive), 28 mai et 4 juin 1954, et 11 mars 1955.

${ }^{18}$ D'après Pieraly Remtoulla, à propos des services administratifs de Majunga pendant la Seconde Guerre mondiale: "Tout le monde est corrompu », entretien, Paris, mai 2007.

${ }^{19}$ Commentaire du haut-commissaire De Chevigné au rapport, Demallle, op. cit. : 30. 
Des fortunes démesurées se créent. D’après Demaille, «une estimation modérée permet d'indiquer qu'une vingtaine d'Hindous ont une fortune supérieure à 50 millions de francs CFA, que plus d'une centaine représentent 10 à 15 millions chacun et qu'une centaine encore disposent en moyenne de 5 millions $^{20}$. »

\section{L'après-guerre}

Les problèmes évoqués ci-dessus, ainsi que le nationalisme malgache et l'indépendance de l'Inde, contribuent à faire du désormais «problème hindou » une question à part entière ${ }^{21}$. Et pourtant il convient de remarquer que l'on assiste dans les années d'après-guerre à l'émergence d'un sentiment métis où l'attraction vers la France se développe. La contribution au développement de la colonie, souvent considérée seulement comme une manœuvre d'enrichissement, est aussi regardée, par ses acteurs, comme une certaine forme d'allégeance. La France attire d'abord pour des raisons d'ordre économique. Les premiers voyages en métropole sont précoces : dès la fin des années 1920 pour H.J. Barday ${ }^{22}$. Mais le processus était à l'œuvre. Il est vrai que la part des Indiens ayant la nationalité française demeure faible. En 1948 moins de 10 \% sont naturalisés ${ }^{23}$. Originaires de l'Inde britannique ils ont, jusqu'à la guerre, conservé leur statut de sujets britanniques. Mais la question de la citoyenneté ne détermine pas la nature des sentiments. Après les voyages d'affaires, la création en France, au lendemain de la guerre, des premiers bureaux d'achats (Remtoullah, Akbaraly Daoudbay, Barday, Hirijee), succursales des sociétés basées à Madagascar, contribuent lentement à transformer la société indienne de Madagascar. Cette étape prépare aussi les suivantes que seront les études en métropole et les migrations à partir des années 1970 pour échapper aux tensions politiques et économiques.

À la fin de la période coloniale c'est Akbaraly Daoudbay, longtemps représentant de la communauté indienne de Madagascar et ayant fait ses études au lycée de Tananarive à partir de 1918, qui crée le premier prix de littérature en langue française de Madagascar. On lui reproche pourtant de ne pas avoir la citoyenneté française. Du point de vue social, les Indiens sont considérés comme inassimilables. Par le mariage endogame, la pratique de la langue gujaratie et l'existence de communautés religieuses spécifiques, ils protègent leurs particularités culturelles. C'est une préoccupation des familles. Mais les efforts actuels pour la préservation de la langue nous obligent à conclure au métissage culturel profond qui s'est opéré pendant le siècle. Dès 1946, Sultan Muhammad Shah, alors chef spirituel et religieux des musulmans shiites ismailis nizarites, pousse ses fidèles à prendre la citoyenneté française. Quelques années plus tard, il entraîne les femmes dans la voie de l'occidentalisation en prônant les modes vestimentaires européens.

\footnotetext{
${ }^{20}$ Demaille, op. cit. : 18.

${ }^{21}$ Dans son commentaire au rapport de l'inspecteur Demaille, P. de Chevigné souligne: «Cette question [celle des Indiens] sera désormais suivie par la Direction des affaires politiques à laquelle des instructions ont été données. ", Demaille, op. cit. : 3.

${ }^{22}$ Mémoires non publiées de Haidaraly Jina Barday, grâce à l'aimable communication de M. Anil Barday.

${ }^{23}$ Demaille, op. cit. : 3.
} 
L'indépendance de l'Inde et la création du Pakistan en 1947 n'ont pas eu les conséquences politiques attendues. Elles ont en réalité, du point de vue identitaire, l'effet inverse. L'administration coloniale tend à surveiller de plus en plus étroitement la minorité indienne. Elle craint en effet qu'elle ne s'érige en un intermédiaire auprès des milieux nationalistes malgaches, de l'idéologie socialiste et anticoloniale du parti de Nehru. Dans l'ensemble, ces faits de l'histoire touchent d'assez loin les communautés gujaraties de l'île. Elles sont attachées à leurs origines et de ce fait restent comme elles le peuvent en contact avec le Gujarat. La presse gujaratie ${ }^{24}$ a toujours circulé et est un moyen d'entretenir des relations avec le pays d'origine. Mais elles commencent à s'estomper et seules certaines personnalités haut placées dans la hiérarchie sociale interne du groupe sont en contact avec le Gujarat, l'Inde et le Pakistan et parfois ses classes dirigeantes ${ }^{25}$. Les événements politiques indiens de l'après-guerre sont une source de fierté indéniable. Mais les communautés commerçantes s'engagent peu sur le terrain politique et se sont souvent les circonstances qui les obligent à prendre parti. Dans ce contexte les positions de la nouvelle ambassade de l'Inde qui tente de diffuser son message politique dérangent plus qu'elles ne rassemblent. Mis à part quelques Hindous qui voient dans l'Inde un gage de sécurité, les autres, même ceux qui pourraient être touchées par des influences nationalistes ${ }^{26}$, sont déjà sur la voie de l'occidentalisation.

\section{Vers la nation malgache?}

Les sentiments à l'égard de Madagascar paradoxalement restent peut-être les moins étudiés. Or malgré la présence française, les questions de nationalités liées à la Grande-Bretagne, l'Inde ou le Pakistan, c'est bien Madagascar qu'il s'agit. Certaines familles étaient présentes avant l'instauration du système colonial. Et pourtant il y a comme un tabou à parler de cette question. Une forme de non-dit perdure du fait de la question économique. Il faut dire que la puissance financière du groupe est inversement proportionnelle à son état numérique ${ }^{27}$. Le processus d'en-

${ }^{24}$ Dans les années 1915-1920, on retrouve à Madagascar des exemplaires de The Gujarati Gujarat mitra et Gujarat Darpau Jaine Fainched Lak Miutre. Source : télégramme officiel du chef de province au gouverneur général au sujet de Gaddar, journal « séditieux » de propagande allemande en langue gujaratie, CAOM, FT/MAD/GGM, 6(2) D25, Nosy-bé, avril 1915.

${ }^{25}$ Par exemple d'après sa nièce, Rawjee a effectivement été en contact avec Nehru.

${ }^{26}$ L'administration coloniale s'inquiète en effet des relations que paraissent entretenir les élites, comme les familles Ismail et Barday, avec l'ambassade de l'Inde. D'après les mémoires de H.J. Barday, les frères de Mamad Ismail vivant au Tanganyika, bien qu'ismaillis, «étaient imprégnés d'une influence hindouiste de la branche Arya Samaj ». Pour les Indiens de Madagascar, les questions nationales, issues de la partition, sont beaucoup moins idéologiques que ne le pensent les autorités coloniales. La direction de la sûreté générale reconnaît d'ailleurs la part des intérêts personnels dans les conflits internes et surtout «une réserve prudente en matière de politique », in Étude sur la colonie indienne de Madagascar, Direction de la Sûreté Générale, Madagascar et dépendances, CAOM, FT/MAD/GGM, 6(1) D91, novembre 1953.

${ }^{27}$ N. Razafimandimby, op. cit. Cinq ans plus tôt, en conclusion d'une étude, on pouvait lire: «Les Indiens aujourd'hui détiennent $41 \%$ de l'économie [...] à l'avenir seront-ils solidaires des Malgaches qu'ils ont souvent négligés et finiront-ils par devenir de véritables nationaux? ", in M. Chandika, Les Indiens à Madagascar, Mahajanga, ms., 1994. 
richissement en temps de crise continue depuis l'indépendance. Des émeutes antiindiennes éclatent comme en 1987, mais la menace ne prend pas la forme des expulsions qui ont existé ailleurs en Afrique de l'Est (Zanzibar en 1964, Ouganda en 1972). Avec plus de virulence, les données restent les mêmes que sous la colonisation. Le pouvoir n'a pas les moyens, quand bien même il le voudrait, de se défaire de cette minorité qui contribue très largement au fonctionnement de l'économie du pays.

Vue sous l'angle politique, la proximité des milieux économiques d'origine indienne avec les leaders politiques n'est pas nouvelle. À la fin des années 1920, Raseta, figure du nationalisme malgache, est le médecin des familles indiennes de Tuléar ${ }^{28}$. Après la Seconde Guerre mondiale, quelques nationalistes sont employés dans les sociétés indiennes. L'administration coloniale suspectait la communauté indienne d'avoir financé, ne serait-ce que faiblement, le mouvement indépendantiste. On sait aussi que sous la Première République (1960-1972) le président Tsiranana invite les Indiens à participer à l'élan de construction nationale. C'est à partir de 1972 que la situation des hommes d'affaires devient problématique. Malgachisation et nationalisation poussent les classes moyennes à émigrer de nouveau vers la France, l'île de la Réunion, le Canada.

Les références au pays abandonné sont nombreuses dans les foyers installés en France. Elles sont parfois plus qu'une simple affection. Elles sont constitutives d'une identité mélangée que l'on redécouvre avec le vécu dans l'ailleurs. On retourne donc voir la famille, les paysages et les saveurs de sa jeunesse. C'est en Malgache, comme certains Indiens restés sur place, qu'ils font leur les traditions et les histoires de la côte ouest. Les croyances et pratiques «païennes » aux yeux d'une certaine orthodoxie religieuse de leur communauté d'origine ne sont pas rares. On consulte le guérisseur, le tromba $a^{29}$ à qui l'on fait des offrandes en gage de bénédictions ${ }^{30}$.

Le métissage, longtemps resté tabou, tend lui aussi à s'officialiser. La perpétuation du mariage endogame reste le grand reproche des Malgaches qui y voient la preuve manifeste d'une volonté de non-intégration. Pourtant eux, qui pendant longtemps ne sont restés que des chotara $^{31}$, commencent à devenir assez nombreux pour qu'on ne puisse désormais éluder leur présence. Les jamatkhana ${ }^{32}$ de Tuléar et de Majunga sont aujourd'hui fréquentés par ces métis qui, quittant les villes et villages de l'arrière-pays, viennent s'installer dans les capitales de provinces.

À la fin de la période coloniale, s'était engagé un processus alors nouveau: celui de l'apparition dans les chambres de commerce, les syndicats et les conseils municipaux, de membres d'origine gujaratie. Cette tendance, témoignant de l'implication des descendants de migrants dans les conseils de représentations et la vie publique, se poursuit aujourd'hui. En haut de la hiérarchie sociale indienne on assiste, en effet, depuis la fin de l'ère Ratsiraka, à l'apparition à la Chambre des

\footnotetext{
${ }^{28}$ Mémoires de H.J. Barday, op. cit.

29 Le terme désigne à la fois le rituel de possession et l'ancêtre, l'esprit possédant.

30 Informations prises aussi auprès des officiants de ce rituel à Majunga en avril 2005.

31 «Bâtard», «mélangé » (avec une connotation péjorative), terme gujarati appliqué à tous les métissages, indo-malgache ou intercommunautaires.

32 Mosquées ismailiennes, littéralement maisons de la communauté.
} 
députés de quelques personnalités d'origine gujaratie: Dramsy et Soundarjee d'Anlalava, Antsohy et Port-Berger dans le nord de Madagascar.

$* * *$

Tenter de retracer l'itinéraire identitaire des Indiens de Madagascar impose la prise en considération d'un certain nombre de facteurs. Le premier bien entendu est le parcours migratoire, et son corollaire le facteur temps. L'étude du processus migratoire nous apprend que plusieurs phases peuvent être dégagées: le voyage saisonnier sur les côtes nord-ouest de Madagascar et son double le voyage d'affaires vers la France moins d'un siècle plus tard; l'installation temporaire pour quelques années (c'est l'époque de la création d'agences, de succursales à la fin du $x^{e}$ siècle et le temps de la création de bureaux d'achats puis des études en France à partir des années 1950) ; enfin, l'installation, pensée définitive, des foyers et des familles. Ce processus, qui peut s'étendre sur trois générations, implique l'existence de personnalités pionnières ouvrant la voie aux autres membres. Et à chaque étape migratoire correspond aussi une certaine forme de sentiments.

Ceci s'observe surtout pour les communautés ayant connu les migrations vers la France, mais pas seulement. Les migrations internationales ont aussi leur pendant aux niveaux national et local. Le départ pour la France des classes moyennes, qui résidaient surtout en ville, a laissé un champ libre et des possibilités nouvelles pour les Indiens les moins aisés restés à Madagascar.

Il serait donc erroné de comprendre les Indiens de Madagascar comme un ensemble monolithique. Leur héritage culturel de communautés d'origine gujaratie et entrepreneuriales contribue à la perpétuation de mécanismes sociaux cohésifs. Mais le politique, au temps de la colonie comme depuis l'indépendance en 1960, a pu contribuer à la construction d'une image de communauté homogène. Or les Indiens, tant par le parcours migratoire que par le niveau socio-économique et la communauté socioreligieuse d'appartenance, n'éprouvent pas tous les mêmes sentiments. Les trois références que sont la communauté, Madagascar et la France ne s'accordent pas toujours de la même façon. En vertu des différences internes évoquées ci-dessus et qui ne sont pas les seules, le genre comme la classe d'âge jouant également un rôle important, chaque individu a son propre ressenti.

La donnée économique et financière liée à celle, avec les indépendances, de la question de l'allégeance à une nation a compliqué le regard et nos tentatives de compréhension du phénomène identitaire. L'incertitude et les sentiments d'insécurité en temps de crise ont contribué aussi, ces dernières années, à l'élaboration de stratégies différentes en fonction des communautés. Densification des relations sociales pour certaines, tentatives d'ouvertures pour d'autres... Ces évolutions récentes ne font pas l'unanimité et sont toujours discutées. Ces amalgames de références identitaires sont le fruit d'un processus historique qui reste en mouvement et évolue. Nous pensons que pour comprendre la réalité des sentiments vécus par les Indiens de Madagascar il faut accepter l'idée d' «identités circonstancielles » formant un ensemble bien articulé et cohérent, mais dont les différents facteurs constitutifs sont mobilisés différemment en fonction des circonstances sociales géographiques et culturelles dans lesquelles l'individu évolue.

Ludovic GANDELOT 